\title{
Delafloxacin: design, development and potential place in therapy
}

\author{
This article was published in the following Dove Press journal: \\ Drug Design, Development and Therapy \\ 20 March 2017 \\ Number of times this article has been viewed
}

\section{Francisco Javier Candel Marina Peñuelas}

Department of Clinical Microbiology and Infectious Diseases, Hospital Clínico San Carlos, Instituto de Investigación Sanitaria San Carlos (IdISSC), Universidad Complutense, Madrid, Spain

\footnotetext{
Correspondence: Francisco Javier Candel Department of Clinical Microbiology and Infectious Diseases, Hospital Clínico San Carlos, Instituto de Investigación Sanitaria San Carlos (IdISSC), Universidad Complutense, Madrid, Spain

Tel +34 913303486

Email fj.candel@gmail.com
}

\begin{abstract}
Delafloxacin (DLX) is a new fluoroquinolone pending approval, which has shown a good in vitro and in vivo activity against major pathogens associated with skin and soft tissue infections and community-acquired respiratory tract infections. DLX also shows good activity against a broad spectrum of microorganisms, including those resistant to other fluoroquinolones, as methicillin-resistant Staphylococcus aureus. Its pharmacokinetic properties and excellent activity in acidic environments make DLX an alternative in the treatment of these and other infections. In this manuscript, a detailed analysis of this new fluoroquinolone is performed, from its chemical structure to its in vivo activity in recently published clinical trials. Its possible place in the current antimicrobial outlook and in other infectious models is also discussed.
\end{abstract}

Keywords: Delafloxacin, fluoroquinolones, methicillin-resistant Staphylococcus aureus, therapy

\section{Introduction}

Complicated skin and soft tissue infections (SSTIs) are common for both outpatient and hospitalized patients and traditionally include various clinical symptoms ranging from minor superficial infections to necrotizing fasciitis with high rates of mortality. Several studies have shown an increase in ambulatory and hospital visits related to these infections, and an increase in length of stay in hospital, mortality risk and health costs. ${ }^{1-3}$ In North American hospitals, an increase of $29 \%$ was detected in hospital admissions because of SSTIs between 2000 and 2004. ${ }^{4}$ In 2010, the Food and Drug Administration (FDA) proposed a new classification, differentiating acute bacterial skin and skin structure infections (ABSSSIs), which include three entities: cellulitis and erysipelas, wound infections and major skin abscesses. Among the involved microorganisms, Staphylococcus aureus is the most common, being the detection of methicillin-resistant $S$. aureus (MRSA) an independent risk factor for increased risk of mortality, length of hospital stay and hospital costs. ${ }^{5}$ Furthermore, S. aureus has a high tolerance to acidic $\mathrm{pH}$, surviving in acidic environments such as abscesses and empyema, where most antibiotics show decreased activity. Gram-negative organisms are isolated in smaller proportion, but the increase of multiresistant bacteria, such as Pseudomonas aeruginosa and beta-lactamase and carbapenemase enterobacteria carriers, has decreased the available therapeutic arsenal. ${ }^{6,7}$

In the field of respiratory infection, pneumonia remains, along with influenza, the respiratory infection with the highest mortality. ${ }^{8}$ Among the most commonly used antibiotics in the treatment of respiratory tract infections (CA-RTIs) are fluoroquinolones (FQ), as well as $\beta$-lactams and macrolides. Despite its still good activity, there has long since been warning about increasing resistance among common pathogens in 
CA-RTIs, such as Streptococcus pneumoniae, Haemophilus influenzae and Moraxella catarrhalis. ${ }^{9,10}$

The resistance of Neisseria gonorrhoeae to quinolones has increased worldwide in the last decade with percentages of $\sim 15 \%-20 \%$, in some geographical areas reaching $50 \%$. ${ }^{11,12}$ Main consequence has been a change in World Health Organization (WHO) recommendations for empiric sexually transmitted infections (STIs) therapy to a cephalosporin and azithromycin combination, reserving the quinolone for targeted therapy. ${ }^{11,13}$

Delafloxacin (DLX) is a new FQ pending approval, which has shown a good in vitro and in vivo activity against major pathogens associated with ABSSSIs and CA-RTIs. It also shows good activity against a broad spectrum of microorganisms, including those resistant to other FQ, and stability against multiresistant strains. Its pharmacokinetic properties and excellent activity in acidic environments make it an alternative in the treatment of these and other infections. In this manuscript, a detailed analysis of this new FQ is performed, from its chemical structure to its in vivo activity in recently published clinical trials. Its possible place in the current antimicrobial outlook and its possible use in other infectious contexts are also discussed.

\section{Fluoroquinolones}

Quinolones are one of the few families of completely synthetic antibiotics, which have allowed many structural modifications on pharmacophore core to increase their potency, enhance their pharmacokinetic properties and reduce their toxicity. FQ arise with the introduction of a fluorine atom in position 6 , improving these described properties. They can act on two bacterial enzymes involved in the replication of genetic material, DNA-gyrase and/or topoisomerase intravenous (IV). In its presence, they form a ternary complex responsible for breaking the double helix of DNA, which blocks replication, causing cell death. There is a relationship between the structure of the quinolone and the preferred activity on the DNA-gyrase or topoisomerase IV. Because of their mechanism of action, they are effective against growing bacteria and in those in stationary phase.

In general, FQ exhibit good gastrointestinal absorption (in the absence of chelators), with good oral bioavailability and a high distribution volume because of their low plasma protein binding, their solubility and ionization characteristics, diffusing well to tissue compartment and into the cell. Although the MICs tend to increase in acidic environments, such as inside phagolysosomes or urine, their preferably renal elimination ensures high urine concentrations, justifying its frequent use in the treatment of urinary tract infections. Because of their broad and high tissue diffusion, FQ are routinely used in the treatment of both community-acquired and nosocomial pneumonia and intra-abdominal infections (IAIs). The pharmacokinetic parameter that best predicts treatment success is area under the curve (AUC)/MIC, considering optimal values $>125 \mathrm{mgh} / \mathrm{L} \cdot{ }^{14,15}$

Bacterial resistance to these antibiotics can have several causes. The first is by modifying the target of action through chromosomal mutations in DNA-gyrase and topoisomerase, resulting in the quinolone resistance-determining region. Second, by reducing the intracellular concentration of the antibiotic, either by reducing the synthesis of porins linked to the penetration of quinolones and other structurally unrelated antibiotics (that occurs mainly in gram-negatives), or by increased expression of active efflux pumps in a dose-dependent manner, associated with a decrease in permeability. Finally, there is a low-level resistance mechanism mediated by PMQR plasmids (plasmidmediated quinolone resistance), which contain genes encoding efflux pumps, protective action target proteins or antibiotic-modifying enzymes derived from aminoglycosidemodifying N-acetyl-transferases (Table 1). ${ }^{16,17}$

\section{Mechanism of action and microbiological activity}

DLX (1[6-amino-3,5-difluoro-2-pyridinyl]-8-chloro-6-fluro7[3-hydroxy-1-azetidinyl]-4-oxo-1,4-dihydroquinoline3-carboxylic acid) has shown higher antibacterial power than other FQ, maintaining the same inhibitory activity of topoisomerase. Its greatest strength seems to derive from three structural differences: ${ }^{18}$ It does not have a strong base in $\mathrm{C} 7$, becoming a weak acid and thus increasing its activity in acidic medium; the chlorine atom in position $\mathrm{C} 8$ acts as an electron-withdrawing group, reducing the reactivity of the heterocycle and stabilizing the molecule; and third, the aromatic ring attached to $\mathrm{N} 1$ increases the molecular surface compared with other quinolones (Figure 1).

By eliminating the basic group in $\mathrm{C} 7$ present in other FQ, DLX loses the ability to act as zwitterion (no-net-charge molecule with two ionized groups) acquiring a weak acid character. Being a weak acid, its pKa (5.4) is lower than other quinolones. As a result, inside of a phagolysosome ( $\mathrm{pH}$ from 5 to 5.5), it will be found primarily in neutral or ionized form, which will facilitate the transmembrane pass into the bacterium through gradient. Once inside the bacteria, where the $\mathrm{pH}$ is neutral, DLX be deprotonated, being, in ionic form, retained within the bacteria. Other FQ, such as 
Table I Main resistance mechanisms in quinolones

\begin{tabular}{ll}
\hline Resistance mechanism & Characteristics \\
\hline $\begin{array}{l}\text { Chromosomal mutations (quinolone resistance- } \\
\text { determining region) }\end{array}$ & $\begin{array}{l}\text { They act over genes encoding the DNA gyrase targets (Gyr A and Gyr B) and topoisomerase IV } \\
\text { (Par C and Par E). They appear for random transcription errors } \\
\text { Reduction of intracellular concentration }\end{array}$ \\
$\begin{array}{ll}\text { Low resistance level } \\
\text { Decrease in drug penetration by reduction of porin expression. It can be selected after } \\
\text { treatment with other antibiotics } \\
\text { Increased expression of active ejection pumps. They are usually linked to the dose of quinolones } \\
\text { Some confer unique resistance to quinolones (NorA-B-C) and others also to structurally } \\
\text { unrelated antibiotics } \\
\text { Coding of active ejection pumps (QacA-B, PmrA) } \\
\text { Encoding proteins in the target of action, decreasing the availability of the target to the } \\
\text { antibiotic } \\
\text { Coding of enzymes modifying the quinolone structure. They are derived from the } \\
\text { aminoglycoside-modifying enzymes }\end{array}$ \\
\hline
\end{tabular}

moxifloxacin (MXF), are presented in an acid medium in the form of zwitterion to a lesser extent than DLX in neutral form, which results in a lower transmembrane passage. With MXF, the majority of the FQ still remain in the zwitterion form within the bacteria, so it is easier to return to the exterior. This explains why DLX MICs can be reduced from 2 to 32 times in acid environments compared with other quinolones. Against $S$. aureus, DLX MICs are between 5 and 7 dilutions lower at $\mathrm{pH} 5.5$ than at $\mathrm{pH} 7.4$, reaching values of $0.00003 \mu \mathrm{g} / \mathrm{mL} .{ }^{19}$ This would justify its good in vivo activity in located infections, usually acidic, such as abscesses and empyema, or acidic anatomical areas such as urine, ${ }^{20}$ the vagina and the stomach. However, one cannot underestimate the possibility that the acidic $\mathrm{pH}$ may affect other transport mechanisms (Figure 2).

In general, the substituents at $\mathrm{C} 7$, and to a lesser extent, $\mathrm{C} 8$, determine the power and the preferential action target of the FQ, that is, the greater or lesser affinity for topoisomerase

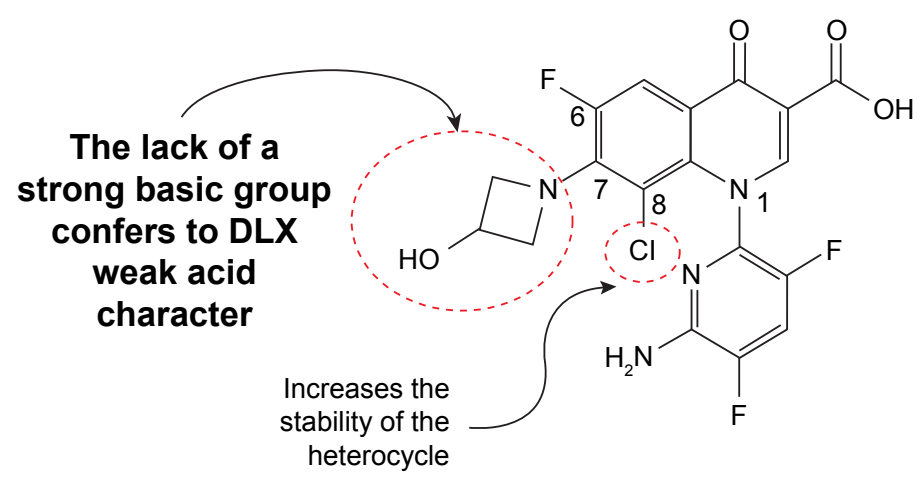

Delafloxacin (DLX)<smiles>C[C@H]1COc2c(N3CCN(C)CC3)c(F)cc3c(=O)c(C(=O)O)cn1c23</smiles>

Levofloxacin (LVX)<smiles>O=C(O)c1cn(C2CC2)c2cc(N3CCNCC3)c(F)cc2c1=O</smiles>

Ciprofloxacin (CPX)<smiles>COc1c(N2CC3CCCNC3C2)c(F)cc2c(=O)c(C(=O)O)cn(C3CC3)c12</smiles>

Moxifloxacin (MXL)

Figure I Chemical structure of DLX compared with other quinolones (LVX, CPX, MXL).

Note: As DLX lacks a protonable substituent group in position 7, it is more acidic than other quinolones.

Abbreviations: CPX, ciprofloxacin; LVX, levofloxacin; MXL, moxifloxacin. 
<smiles>Nc1nc(-n2cc(C(=O)O)c(=O)c3cc(F)c(N4CC(O)C4)c(Cl)c32)c(F)cc1F</smiles>

Neutral form<smiles>Nc1nc(-n2cc(C(=O)O)c(=O)c3cc(F)c(N4CC(O)C4)c(Cl)c32)c(F)cc1F</smiles>

Anionic form

(DLX)

Predominant form at acidic $\mathrm{pH}$
Predominant form at physiological pH

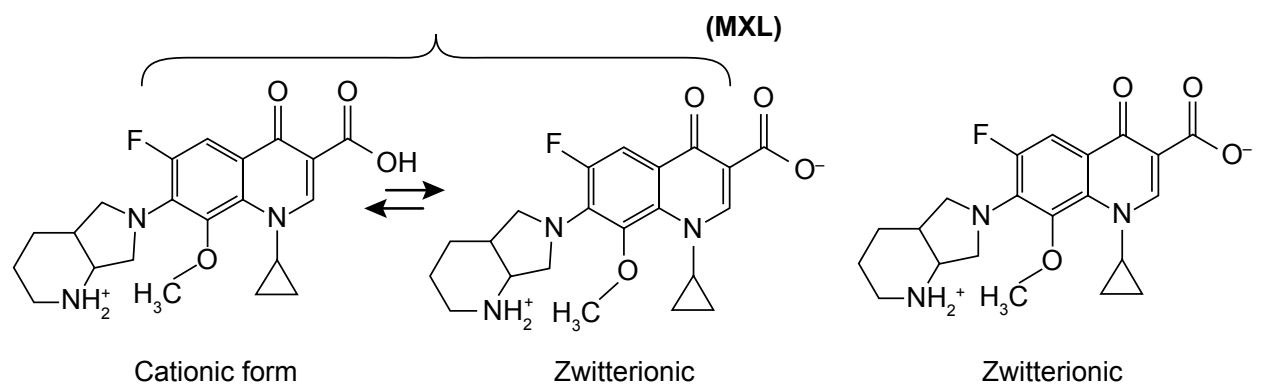

Figure 2 Predominant form of DLX (top) and MXL (bottom) at acidic and neutral pH.

Notes: The absence of a basic group in $\mathrm{C7}$ allows $\mathrm{DLX}$ to be neutral at acidic $\mathrm{pH}$ and anionic at physiological pH. The opposite happens with MXL. Abbreviations: DLX, delafloxacin; MXL, moxifloxacin.

IV or gyrase. DLX shows a similar affinity for both targets, which explains its greater stability against such modifications, as DLX resistance requires the accumulation of several mutations that collectively affect both targets. Moreover, DLX does not seem to be a good substrate for efflux pumps. For this, coupled with its high power, it can maintain lower MICs despite this expression and other resistance mechanisms. ${ }^{21}$ In fact, the concentration of antibiotics necessary to prevent the selection of resistant mutants is between one and four times the MIC, resulting in very low values, lower than those of any other FQ. ${ }^{22}$

DLX has shown excellent activity against gram-positives (S. aureus, S. pneumoniae, B-hemolytic Streptococci, some Enterococcus sp), gram-negatives (H. influenzae, M. catarrhalis, N. meningitidis, N. gonorrhoeae, Enterobateriaceae, Pseudomonas spp), anaerobes (Bacteroides sp, Prevotella sp, Clostridium difficile, Clostridium perfringens), intracellular microorganisms (Mycoplasma sp, Ureaplasma sp, Chalmydia $\mathrm{sp}$ ) and M. tuberculosis (Table 2). ${ }^{18,19,21-31}$

Regarding the biofilm activity, an in vitro study comparing several antibiotics against biofilm produced by methicillin-susceptible and -resistant $S$. aureus strains (MSSA and MRSA), DLX and daptomycin were the most effective antibiotics in clinical use concentrations, obtaining in both cases a reduction of bacterial viability exceeding $50 \%{ }^{32}$ DLX penetration into the biofilm is inversely proportional to the thickness of the polysaccharide, varying between $0.6 \%$ and $52 \%$, and its activity is the highest on biofilms with an acid microenvironment, which seems to occur frequently. ${ }^{33}$

Against $N$. gonorrhoeae, in vitro studies on strains sensitive and resistant to ciprofloxacin (CPX) obtained excellent efficacy results with an $\mathrm{MIC}_{50}$ of $0.06 \mu \mathrm{g} / \mathrm{mL}$ and $\mathrm{MIC}_{90}$ of $0.125 \mu \mathrm{g} / \mathrm{mL}$. The frequency of spontaneous mutations was $\leq 10^{-7}$, maintaining good stability in the study of the selection of resistant mutants through daily passes over media with subinhibitory concentrations of DLX. ${ }^{34}$

\section{Pharmacokinetics and metabolism}

There are several studies with DLX, dedicated to pharmacokinetic properties, tolerability, effect of concomitant food administration and safety. ${ }^{35,36}$ After endovenous administration, ${ }^{35}$ the value of maximum concentration $\left(\mathrm{C}_{\max }\right)$ increases proportionally with increasing doses within the range from 300 to $1,200 \mathrm{mg}$. However, the AUC increases more than proportionally with the dose in the same range. The cause of this lack of proportionality has not yet been 
Table 2 In vitro activities among different quinolones

\begin{tabular}{|c|c|c|c|c|c|c|}
\hline Organism & No of strains & Antibacterial agents & MIC range & $\mathrm{MIC}_{50}$ & $\mathrm{MIC}_{90}$ & Ref \\
\hline \multirow[t]{5}{*}{ Staphylococcus aureus FQ-S } & 70 & LVX & $0.06-0.5$ & 0.25 & 0.5 & 25 \\
\hline & 88 & LVX & $0.06-1$ & 0.12 & 0.25 & 26 \\
\hline & 70 & $M X L$ & $0.015-0.5$ & 0.06 & 0.1 & 25 \\
\hline & 70 & DLX & $0.002-0.008$ & 0.004 & 0.008 & 25 \\
\hline & 88 & DLX & $\leq 0.001-0.06$ & 0.002 & 0.008 & 26 \\
\hline \multirow[t]{5}{*}{ S. aureus FQ-R } & 71 & LVX & $4-64$ & 16 & 32 & 25 \\
\hline & 100 & LVX & $2-32$ & 4 & 8 & 26 \\
\hline & 71 & $M X L$ & $0.25-16$ & 4 & 8 & 25 \\
\hline & 71 & DLX & $0.015-1$ & 0.25 & I & 25 \\
\hline & 100 & DLX & $0.015-2$ & 0.006 & 0.12 & 26 \\
\hline \multirow[t]{2}{*}{ Coagulase-negative staphylococci } & 19 & LVX & $0.06->32$ & 0.12 & $>32$ & 26 \\
\hline & 19 & DLX & $0.00 I-2$ & 0.004 & I & 26 \\
\hline \multirow[t]{3}{*}{ S. pneumoniae FQ-S } & 69 & LVX & $0.5-2$ & I & I & 25 \\
\hline & 69 & $M X L$ & $0.06-0.25$ & 0.12 & 0.12 & 25 \\
\hline & 69 & DLX & $0.004-0.015$ & 0.008 & 0.015 & 25 \\
\hline \multirow[t]{3}{*}{ S. pneumoniae FQ-R } & 33 & LVX & $2-32$ & 16 & 32 & 25 \\
\hline & 33 & $M X L$ & $0.25-8$ & 2 & 4 & 25 \\
\hline & 33 & DLX & $0.015-0.5$ & 0.12 & 0.5 & 25 \\
\hline \multirow[t]{2}{*}{ Neisseria gonorrhoeae } & 100 & $\mathrm{CPX}$ & $\leq 0.015-16$ & $\leq 0.015$ & 8 & 27 \\
\hline & 100 & DLX & $\leq 0.0005-0.06$ & 0.001 & 0.06 & 27 \\
\hline \multirow[t]{3}{*}{ Enterococcus faecium FQ-R } & 28 & LVX & $8->128$ & 32 & 64 & 25 \\
\hline & 28 & $M X L$ & $1-32$ & 16 & 16 & 25 \\
\hline & 28 & DLX & $0.25-16$ & 4 & 8 & 25 \\
\hline \multirow[t]{2}{*}{ Escherichia coli FQ-R } & 27 & CPX & $4->128$ & 128 & $>128$ & 25 \\
\hline & 27 & DLX & $1-16$ & 4 & 8 & 25 \\
\hline \multirow[t]{2}{*}{ Klebsiella pneumoniae FQ-R } & 22 & CPX & $4-64$ & 32 & 64 & 25 \\
\hline & 22 & DLX & $1-4$ & 2 & 4 & 25 \\
\hline \multirow[t]{2}{*}{ Bacteroides fragilis } & 16 & LVX & $\mathrm{I}-2$ & 2 & 2 & 23 \\
\hline & 16 & DLX & $0.03-0.12$ & 0.06 & 0.12 & 23 \\
\hline \multirow[t]{2}{*}{ Pseudomonas aeruginosa FQ-R } & 21 & $\mathrm{CPX}$ & $2-128$ & 32 & 128 & 25 \\
\hline & 21 & DLX & $1-128$ & 32 & 128 & 25 \\
\hline
\end{tabular}

Abbreviations: CPX, ciprofloxacin; DLX, delafloxacin; FQ, fluoroquinolone; LVX, levofloxacin; MXL, moxifloxacin.

clarified, but it has been linked to the saturation of the elimination routes. The half-life $\left(t_{1 / 2}\right)$ varies in a range of $\sim 8 \mathrm{~h}$, with doses of $300 \mathrm{mg}$, up to $17 \mathrm{~h}$ with higher doses, exhibiting a biexponential decrease in the plasma concentration. DLX shows good distribution, with a volume of distribution (Vd) at a steady state of $35 \mathrm{~L}$ (range 30.21-38.46 L), similar to the total water volume of the body. DLX excretion is predominantly renal (65\%) and mainly in unchanged form, with $<20 \%$ of the initial dose as glucuronide derivatives, recovering $28 \%$ of the total dose in feces. DLX clearance is reduced in patients with moderate and severe renal impairment. However, after 14 days of IV treatment with two daily doses, there is no drug accumulation detected, and clearance on day 14 was similar to day 1 .

The oral bioavailability is $58.8 \%$, with $450 \mathrm{mg}$ taken orally being equivalent to IV infusion of $300 \mathrm{mg}$ for $1 \mathrm{~h}$, permitting oral sequencing. ${ }^{36}$ Similarly, the $\mathrm{C}_{\max }$ and AUC increase in the dose was proportional or higher. The steady state was reached on the third day with minimal drug accumulation. Administered in tablet form along with food, the $\mathrm{C}_{\max }$ scarcely decreased without affecting the AUC, justifying its administration with food, because the effectiveness of the FQ is linked to the total exposure (AUC) and not the peak concentration $\left(\mathrm{C}_{\max }\right)$. In adults aged $>65$ years, the values of $\mathrm{C}_{\max }$ and AUC were $35 \%$ higher than those observed in young adults, in part due to the decreased renal clearance in the elderly population. In several patients, secondary plasma peaks were detected that were attributed to the enterohepatic cycle. With all these, the recommended dosage for IV use is $300 \mathrm{mg}$ every $12 \mathrm{~h}$, and orally, $450 \mathrm{mg}$ every $12 \mathrm{~h}$.

The parameter of effective dosage that best defines the fluoroquinolones is AUC/MIC, being recommended a greater ratio than 60 against gram-positives and $>125$ against gram-negatives ( $>250$ to avoid the development of resistant mutants). ${ }^{14,15}$ In animal models, DLX has been shown to reach these parameters against $S$. aureus, $S$. pneumoniae, Escherichia coli and $P$. aeruginosa. ${ }^{21,37-39}$ 


\section{Efficacy}

\section{Animal models}

A mouse study evaluated the effectiveness of treatment with $300 \mathrm{mg}$ intravenously or $450 \mathrm{mg}$ orally twice daily in lung infection models produced by inoculation of $50 \mathrm{mg}$ with an approximate concentration of $10^{8} \mathrm{CFU} / \mathrm{mL}$ of $S$. aureus (one sensitive and four resistant strains to methicillin), S. pneumoniae (one sensitive and three resistant strains to penicillin) and Klebsiella pneumoniae (one strain sensitive to beta-lactams and three extended spectrum beta-lactamase carriers). The results confirm that the pharmacokinetic/pharmacodynamic (PK/PD) parameter that best predicted the therapeutic efficacy was AUC/CMI, and conclude that with the described doses, DLX would a good therapeutic option for the treatment of pneumonia by $S$. aureus, including the methicillin-resistant $S$. pneumoniae and the penicillin-resistant $K$. pneumoniae including the ESBL strains. ${ }^{40}$ Another study on murine thigh infection model concluded that the pharmacokinetic parameter that best predicts the clinical response is AUC/MIC. ${ }^{41}$

\section{Clinical trials}

A phase II study compared two doses of DLX (300 mg/12 h and $450 \mathrm{mg} / 12 \mathrm{~h}$ intravenously) with tigecycline (TIG) (100 mg intravenously loaded followed by $50 \mathrm{mg} / 12 \mathrm{~h}$ ) for 5-14 days in 150 patients with complicated SSTI (36\% cellulitis, 33\% abscess and 31\% wound infection). They evaluated the clinical response and effectiveness at 14-21 days after the last dose. MRSA was isolated in more than two-thirds of the cases, predominating the Gram-positive cocci. The cure rate was $>90 \%$ in all three groups. The best-tolerated DLX regimen was $300 \mathrm{mg}$ intravenously two times a day. Main $\mathrm{CMI}_{90}$ in S. aureus group was $0.06 \mu \mathrm{g} / \mathrm{mL}$ for DLX, $0.12 \mu \mathrm{g} / \mathrm{mL}$ for $\mathrm{TIG}, 4 \mu \mathrm{g} / \mathrm{mL}$ for $\mathrm{LVF}$ and $8 \mu \mathrm{g} / \mathrm{mL}$ for CPX. ${ }^{42}$

Regarding the SSTIs, another phase II study evaluated the efficacy of endovenous treatment with DLX, linezolid and vancomycin in 256 patients with SSTIs (45\% cellulitis, $28.5 \%$ abscesses, $25 \%$ wound infection and $1.5 \%$ burns). A good clinical response with total resolution of the signs and symptoms of infection is taken as the first end point. ${ }^{43}$ S. aureus was the most isolated microorganism (91\%) among patients with positive cultures, with more than half being methicillin-resistant. DLX reached the highest cure rate with no significant differences with respect to linezolid, but with vancomycin. It seems to have been influenced, in part, by better results in obese patients (BMI $>30 \mathrm{~kg} / \mathrm{m}^{2} ; 78.8 \% \mathrm{DLX}$ vs $58.8 \%$ linezolid vs $48.8 \%$ vancomycin, $P<0.05$ ).

According to the preliminary data provided by pharmaceutical company that markets DLX, in relation to the two phase III studies used for FDA approval, at doses of $300 \mathrm{mg}$ endovenous td and $450 \mathrm{mg}$ orally td, DLX showed no inferiority to the combination of aztreonam and vancomycin in the treatment of complicated SSTI (with two or more signs of systemic infection). ${ }^{44,45} \mathrm{Six}$ hundred and sixty and 860 patients participated in those studies, respectively. In both, the primary European Medicines Agency end point was the reduction of lesion size in the first 48-72 $\mathrm{h}$ and the clinical response at 28 days.

Regarding the respiratory infection, a phase II study concluded that the effectiveness of DLX (200 mg each day for 5 days) was equivalent to that of LVX (500 mg daily for 7 days) in the treatment of acute exacerbation of chronic obstructive pulmonary disease (COPD). ${ }^{46}$ Recently, a phase III study has begun that aims to evaluate, on 860 patients, the clinical efficiency and safety of DLX in comparison with MXF, or linezolid in the case of MRSA, in the treatment of the community-acquired pneumonia. The estimated completion date for the study is July 2018. ${ }^{47}$

By mid-2017, data are expected to be published from a phase III study evaluating the microbiological response and resolution of symptoms of uncomplicated urogenital gonorrhea, and secondly, other anatomical locations, with DLX doses of $450 \mathrm{mg}$ td orally, using intramuscular injection of $250 \mathrm{mg}$ ceftriaxone as a comparator. ${ }^{48}$

\section{Safety and tolerability}

Several studies have shown that the incidence of adverse effects (AE) with DLX is dependent on the dose. ${ }^{46}$ In the phase I study of dose escalation, ${ }^{35}$ adverse reactions were detected in half or more of the individuals who received a dose of $800 \mathrm{mg}$ or more, while with doses of $300 \mathrm{mg}$, the AE detected were equivalent to those of the placebo. In the study with oral administration, ${ }^{36}$ DLX was well tolerated in the range of doses tested (50-1,600 mg). Both by intravenously and orally, the AE were predominantly gastrointestinal (diarrhea of low to moderate intensity) and mainly in high doses, starting from $800 \mathrm{mg}$. In the multiple-dose study, an increase of alanine aminotransferase (ALT) was detected in seven of the 40 participants, two of them reaching values more than two times over the normal limits, without getting a link clear with the increase in the dose.

In the phase II study that compared two doses of DLX with $\mathrm{TIG}^{42}$ the patients who received DLX orally suffered fewer adverse reactions in comparison with the other two branches, because among the main adverse reactions found were, besides the gastrointestinal, those related to IV infusion. The highest rate of adverse reactions was 
obtained on the TIG branch. A patient who received DLX through IV suffered an adverse reaction by hypoglycemia that is linked to the antibiotic, and in several patients of the two DLX groups, a descent of the glucose plasma levels was detected.

In a comparative study of DLX, linezolid and vancomycin treating SSTIs under 14 days, ${ }^{43}$ the group that received DLX reported the highest number of AE (74.4\% for DLX, $72 \%$ linezolid and $64.6 \%$ vancomycin), mostly digestive (nausea being the most frequent). Two cases of elevation of the ALT and AST levels were also detected, probably related to the drug, one in the DLX group and one in the vancomycin group. No AE were reported associated with hypoglycemia.

In the phase III studies that compared the efficacy and safety of IV and oral DLX with respect to the combination of aztreonam and vancomycin, DLX showed good tolerance, with $0.8 \%$ of withdrawals because of AE related to treatment. ${ }^{44,45}$

Due to its predominantly renal elimination, a phase II study evaluated the safety of DLX in individuals with several degrees of renal failure, concluding with the recommendation of lowering the DLX IV dose to $200 \mathrm{mg}$ when the glomerular filtration rate is under $30 \mathrm{~mL} / \mathrm{min}^{4}{ }^{49}$

In a recent phase I study, the safety of a single IV dose of 300 mg of DLX was evaluated in individuals with mild, moderate and severe hepatic impairment (Child-Pugh A, B and C, respectively) comparing the data with those with healthy controls. ${ }^{50}$ Both the AUC and the $\mathrm{C}_{\max }$ and the clearance did not vary significantly in either the liver failure group or the control group. Based on these results, the authors suggest that it would not be necessary to adjust the dose in patients with liver failure.

In no safety study to date there have been reported cases of AE of diarrhea due to $C$. difficile, which could partly be explained because of the anaerobicida activity of DLX with low MICs $(<0.015 \mu \mathrm{g} / \mathrm{mL})$ in vitro against this pathogen. ${ }^{28}$ However, and in spite of the double target that makes difficult the selection of resistant mutants and its good activity against MRSA, beta-lactamase-producing enterobacteria (ESBL) and fluoroquinolone-resistant isolates, we must not forget the close relationship between the massive use of fluoroquinolones and the selection of specific clones of MRSA, ESBL and ribotype 027 C. difficile strains. ${ }^{51}$ In the absence of clinical and epidemiological data that can only be obtained once the antibiotic is marketed, the most reasonable approach is to be cautious and avoid indiscriminate use.

In our review we found no evidence of $\mathrm{AE}$ at the level of the tendons, muscles, joints, nerves and central nervous system. However, the recent FDA warning makes its surveillance convenient. ${ }^{52}$

\section{Potential place in therapy: rationale for clinical indications}

Quinolones meet a series of pharmacokinetic, pharmacodynamic and in vitro activity characteristics, making them attractive for prescription. They are fat-soluble drugs, have a high volume of distribution and tissue affinity, good bioavailability and endovenous-oral correlation near 100\%, in addition to being bactericidal. The choice of one or the other depends on the potential metabolic interaction and the microbiological spectrum that we wish to achieve for each infection model. While all have a broad spectrum against gram-positives and gram-negatives, there are quinolones with more antipseudomonal activity (CPX, LVX), others more active against Staphylococcus spp and S. pneumoniae (LVX, MXL, DLX) and others with activity against anaerobes (MXL, DLX). Looking at metabolisms, there are quinolones with hepatic metabolism and high affinity for the cytochrome P450 system (CPX), and others with low or no affinity for the human hepatic microsomal systems, as they are removed unchanged, mostly through urine (DLX). Taking these factors into account, the choice of the quinolone depends on the infection model, patient comorbidity and the expected or isolated flora. Table 3 summarizes the rationale for clinical indications of DLX.

In respiratory infections, the possible use of DLX in COPD exacerbations has been validated in a recent study comparing it with LVX. ${ }^{46}$ An interesting study is ongoing against MXL in the empiric therapy of community-acquired pneumonia, with both quinolones having a better microbiological profile against respiratory pathogens. Given the activity of DLX against MRSA, the study considers linezolid as a comparator if this pathogen is isolated. ${ }^{47}$ The activity

Table 3 Potential place in therapy of delafloxacin

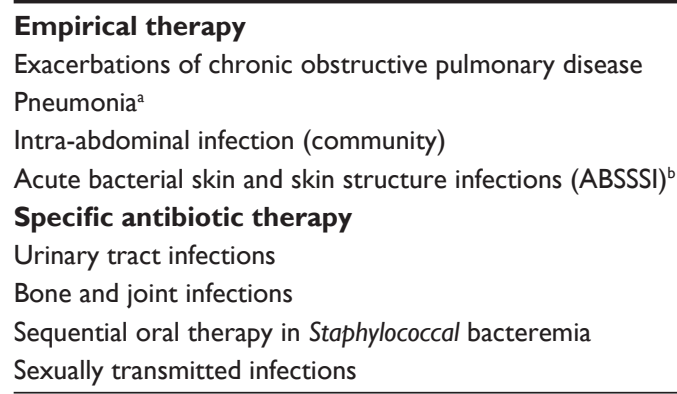

Notes: a Community-acquired pneumonia, hospital-acquired pneumonia and aspirative

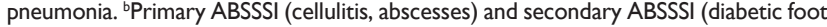
infections, surgical site infections, burns). 
against MRSA, and against enterobacterias, makes DLX a reasonable alternative in the treatment of nosocomial pneumonia. In addition, in murine experimental models, DLX showed high penetration in the pulmonary compartment, ${ }^{40}$ and higher concentrations of free antibiotic in the epithelial lining fluid than in the plasma. ${ }^{53}$ The loss of $\mathrm{C} 7$ in its chemical structure, and with it, the ability to act as zwitterion, facilitates its in vivo activity in acidic mediums. This, coupled with the in vitro sensitivity profile against anaerobes, ${ }^{23}$ its lipid solubility and pulmonary diffusion, could justify its use in the empirical treatment of aspiration pneumonia (like its predecessor, MXL) or bacterial pulmonary abscesses.

The IAI is another infection model requiring the aforementioned PK/PD characteristics, and activity against aerobic and anaerobic mixed flora. MXL, which is the quinolone that most resembles DLX, presents an optimal penetration in the peritoneal exudate of the patient with peritonitis, maintaining concentrations above the MIC of the pathogen isolated in this infection model. ${ }^{54}$ In a randomized study of 656 patients with IAI, MXL was noninferior to PP/TZ, ${ }^{55}$ and in another, no less than the combination of ceftriaxone and metronidazole. ${ }^{56}$ In the four most important monotherapy studies with MXL in IAI, of the 642 anaerobic isolates, 561 (87.4\%) were sensitive with an MIC $\leq 2 \mathrm{mg} / \mathrm{L}, 34$ (5.3\%) were intermediate with MIC of $4 \mathrm{mg} / \mathrm{L}$ and 47 (7.3\%) were resistant, with $\mathrm{MICs} \geq 8 \mathrm{mg} / \mathrm{L} .{ }^{57} \mathrm{DLX}$ has a microbiological profile similar to MXL against enterobacteriaceae and anaerobes and a favorable pharmacodynamic profile for this infection model because of its lipid solubility and diffusion in acid mediums. This would put it in a potential utility in empirical therapy for this type of infection. Finally, the in vitro activity of DLX against Helicobacter pylori ${ }^{28}$ and its favorable behavior in acid media make this drug an attractive alternative in the salvage therapy of this infection because of the increase of resistance in standard therapy (macrolides, amoxicillin, metronidazole, rifamycins).

DLX has renal excretion, with $65 \%$ in unaltered form. The high concentration in the urinary tract of quinolones and the diffusion to prostate tissue ${ }^{58,59}$ could make DLX an attractive alternative for the targeted therapy toward prostatitis and orchiepididymitis because of susceptible pathogens.

SSTI is the model in which there is more scientific evidence with DLX and in which empiric therapy is more attractive in both primary infections (cellulitis, abscesses) and secondary (surgical wound infections, burns and especially diabetic foot infections [DFI]). In two phase II clinical trials, in comparison with linezolid, TIG or vancomycin, the DLX clinical response was significantly greater than vancomycin $(70.4 \%$ vs $54.1 \%, P<0.031)$ and greater, although not significantly, than linezolid (70.4\% vs $64.9 \%$, $P<0.4)$. Regarding TIG, there was no difference $(94.3 \%$ vs $91.2 \%, P<0.6)$. In post hoc analysis, the percentage of patients cured in the DLX group was significantly higher than that in vancomycin in obese patients $(78.8 \%$ vs $48.8 \%$, CI $(-50.7,-9.3), P<0.009){ }^{43}$

DLX has the tissue affinity of an oxazolidinone or a glycylcycline, as both present activity against MRSA and it is also a bactericidal antibiotic. These features are especially needed in DFI. There is evidence of MXL utility in the treatment of DFI, with similar results to piperacillin-tazobactam followed by amoxicillin-clavulanate, even in patients with major vascular involvement (ischemic) or structural (large or profound ulcers), ${ }^{60-62}$ because it keeps antimicrobial concentrations in the perinecrotic tissue above the MIC. ${ }^{63}$ DLX provides over MXL the activity against MRSA and its diffusion in acid mediums, as is frequent in diabetic foot ischemia.

Another important feature of FQ, linking the DFI with osteoarticular infection, is bone penetration. Therefore, quinolones have been widely used in the treatment of osteomyelitis and infections in osteosynthesis materials. ${ }^{64}$ The bone concentrations of FQ in patients undergoing hip or knee arthroplasty exceed $50 \%$ of the plasma concentration, and are higher than the MICs of the microorganisms causing the infection. ${ }^{65-67}$ In addition to bone diffusion, in implant-related infections, the antimicrobial diffusion through the biofilm is important. Regarding this, experimental models, with biofilms of $1 \%$ alginate, demonstrated that CPX and LVX diffuse $\sim 60 \%$, a figure lower than ceftazidime ( $80 \%$ ) and much higher than the aminoglycosides (gentamicin $<14 \%) .{ }^{68}$ The inhibitory activity of MXL, at concentrations of $0.5 \mathrm{mg} / \mathrm{L}$, on the biofilm of $S$. aureus is $\sim 70 \% .{ }^{69}$ Two recent in vitro studies show that DLX is more active removing viable bacteria and stays longer in the thickness of the mature biofilm than MXL against MSSA and with an activity comparable only to daptomycin against MRSA. ${ }^{32,33}$ The bone diffusion of DLX, the diffusion in the thickness of the mature MRSA biofilm, and its oral bioavailability would make the molecule an elective alternative in targeted therapy at osteoarticular infections.

DLX is very active against intracellular microorganisms responsible for STIs (Chlamydia trachomatis, Ureaplasma urealyticum, Mycoplasma hominis), while maintaining in vitro between 2 and 4 times more activity than CPX. ${ }^{27,28}$ On the other hand, it presents strong activity against $N$. gonorrhoeae with distributions of $\operatorname{MIC}(0,001-0,125)$, even in strains resistant to CPX (CMI 2-16 mg/L). ${ }^{34}$ As discussed above, the resistance of $N$. gonorrhoeae to FQ has increased worldwide in the last decade with percentages 
of $\sim 15-20$, in some geographical areas reaching $50,,^{11,12}$ and consequently, the WHO has modified the recommendations in empiric therapy of STDs to combinations of cephalosporin and azithromycin. ${ }^{11,13}$ However, DLX could be useful in directed therapy against sensitive strains.

In relation to the metabolism of FQ, the DLX characteristics can also help optimize antimicrobial treatment. DLX is excreted renally, without having any interaction with the cytochrome P450 system or any human hepatic microsomal system. ${ }^{50}$ This condition makes it a useful drug in the treatment of infections in patients concurrently treated with drugs metabolized by this route (calcineurin inhibitors, antiretroviral agents, metabolic inducers, and so on). Thus, it could be useful in therapy targeted at tuberculosis infection in rifampicin-free schemes, in a similar way to how MXL is used.

Finally, and in relation to sequential therapy and shortening of admission, DLX has enough advantages in terms of oral bioavailability, bactericidal potency and microbiological activity to be considered as an oral alternative, after reaching clinical stability in the home treatment of the bacteremia because of MSSA and MRSA.

All these hypothetical infection models that could have DLX utility should be validated by specific clinical studies. Some of these are ongoing yet or have already been validated.

\section{Disclosure}

The authors declare no conflicts of interest in this work and have not received financial support.

\section{References}

1. Hatoum HT, Akhras KS, Lin SJ. The attributable clinical and economic burden of skin and skin structure infections in hospitalized patients: a matched cohort study. Diagn Microbiol Infect Dis. 2009; 64(3):305-310.

2. Engemann JJ, Carmeli Y, Cosgrove SE, et al. Adverse clinical and economic outcomes attributable to methicillin resistance among patients with Staphylococcus aureus surgical site infection. Clin Infect Dis. 2003;36(5):592-598.

3. Itani KMF, Merchant S, Lin SJ, Akhras K, Alandete JC, Hatoum HT. Outcomes and management costs in patients hospitalized for skin and skin-structure infections. Am J Infect Control. 2011;39(1):42-49.

4. Edelsberg J, Taneja C, Zervos M, et al. Trends in US hospital admissions for skin and soft tissue infections. Emerg Infect Dis. 2009;15(9): 1516-1518.

5. Amin AN, Cerceo EA, Deitelzweig SB, Pile JC, Rosenberg DJ, Sherman BM.Hospitalist perspective on the treatment of skin and soft tissue infections. Mayo Clin Proc. 2014;89(10):1436-1451.

6. Bassetti M, Della Siega P, Pecori D, Scarparo C, Righi E. Delafloxacin for the treatment of respiratory and skin infections. Expert Opin Investig Drugs. 2015;24(3):433-442.

7. Boucher HW, Talbot GH, Bradley JS, et al. Bad bugs, no drugs: no ESKAPE! An update from the Infectious Diseases Society of America. Clin Infect Dis. 2009;48(1):1-12.

8. File TM, Marrie TJ. Burden of community-acquired pneumonia in North America adults. Postgrad Med. 2010;122(2):130-141.
9. Doern GV, Heilmann KP, Huynh HK, Rhomberg PR, Coffman SL, Brueggemann AB. Antimicrobial resistance among clinical isolates of Streptococcus pneumonia in the United States during 1999-2000, including a comparison of resistance rates since 1994-1995. Antimicrob Agents Chemother. 2001;45(6):1721-1729.

10. Hoban Dj, Doern GV, Fluit AC, et al. Worldwide prevalence of antimicrobial resistance in Streptococcus pneumonia, Haemophilus influenza, and Moraxella catarrhalis in the SENTRY Antimicrobial Surveillance Program, 1997-1999. Clin Infect Dis. 2001;32(Suppl 2):S81-S93.

11. WHO. Guidelines for the Ttreatment of Neisseria Gonorrhoeae. Geneva: WHO; 2016. ISBN-13:978-92-4-154969-1.

12. Metha SD, Maclean I, Ndinya-Achola JO, et al. Emergence of quinolone resistance and cephalosporin MIC creep in Neisseria gonorrhoeae Isolates from a cohort of young men in kisumu, Kenya, 2002 to 2009. Antimicrob Agents Chemother. 2011;55(8):3882-3888.

13. Unemo M. Current and future antimicrobial treatment of gonorrhoea the rapidly evolving Neisseria gonorrhoeae continues to challenge. BMC Infectious Diseases. 2015;15:364.

14. Nakamura T, Shimizu C, Kasahara M, et al. Monte Carlo simulation for evaluation of the efficacy of carbapenems and new quinolones against ESBL-producing Escherichia coli. J Infect Chermother. 2009; 15(1):13-17.

15. Moczygemba LR, Frei CR, Burgess DS. Pharmacodynamic modeling of carbapenems and fluoroquinolones against bacteria that produce extended-spectrum beta-lactamases. Clin Ther. 2004;26(11): 1800-1807.

16. Röderova M, Halova D, Papousek I, et al. Characteristics of Quinolone Resistance in Escherichia coli Isolates from Humans, Animals, and the Environment in the Czech Republic. Front Microbiol. 2016;7:2147.

17. Tayebi Z, Heidari H, Kazemian H, Ghafoori SM, Boroumandi S, Houri H. Comparison of quinolone and beta-lactam resistance among Escherichia coli strains isolated from urinary tract infections. Infez Med. 2016;24(4):326-330.

18. Kocsis B, Domokos J, Szabo D. Chemical structure and pharmacokinetics of novel quinolone agents represented by avarofloxacin, delafloxacin, finafloxacin, zabofloxacin and nemonoxacin. Ann Clin Microbiol Antimicrob. 2016;15(1):34.

19. Lemaire S, Tulkens PM, Van Bambeke F. Contrasting effects of acidic $\mathrm{pH}$ on the extracellular and intracellular activities of the antiGram-positive fluoroquinolones moxifloxacin and delafloxacin against S. aureus. Antimicrob Agents Chemother. 2011;55(2):649-658.

20. So W, Crandon JL, Nicolau DP. Effects or Urine Matrix and $\mathrm{pH}$ on the Potency of Delafloxacin and Ciprofloxacin against Urogenic Escherichia coli and Klebsiella penuemoniae. J Urol. 2015;194(2):563-570.

21. Van Bambeke F. Delafloxacin, a non-zwitterionic fluoroquinolone in Phase III of clinical development: evaluation of its pharmacology, pharmacokinetics, pharmacodynamics and clinical efficacy. Future Microbiol. 2015;10(7):1111-1123.

22. Remy JM, Tow-keogh CA, McConnell TS, Dalton JM, Devito JA. Activity of delafloxacin against methicillin-resistant Staphylococcus aureus: resistance selection and characterization. J Antimicrob Chemother. 2012;64(12):2814-2820.

23. Goldstein EJC, Citron DM, Merriam CV, Warren YA, Tyrrell KL, Fernandez HT. In vitro activities of ABT-492, a new fluoroquinolone, against 155 aerobic and 171 anaerobic pathogens isolated from antral sinus puncture specimens from patients with sinusitis. Antimicrob Agents Chemother. 2003;47(9):3008-3011.

24. Harnett SJ, Fraise AP, Andrews JM, Jevons G, Brenwald NP, Wise R. Comparative study of the in vitro activity of a new fluoroquinolone, ABT-492. J Antimicrob Chemother. 2004;53(5):783-792.

25. Zhanel GG, Palatnick L, Nichol KA, Low DE, Hoban DJ; CROSS Study Group. Antimicrobial resistance in Haemophilus influenza and Moraxella catarrhalis respiratory tract isolates: results of the Canadian Respiratory Organism Susceptibility Study, 1997 to 2002. Antimicrob Agents Chemother. 2003;47(6):1875-1881.

26. Hammerschlag MR, Roblin PM. The in vitro activity of a new fluoroquinolone, ABT-492, against recent clinical isolates of Chlamydia pneumonia. J Antimicrob Chemother. 2004;54(1):281-282. 
27. Waites KB, Crabb DM, Duffy LB. Comparative in vitro susceptibilities and bactericidal activities of investigational fluoroquinolone ABT-492 and other antimicrobial agents against human mycoplasmas and ureaplasmas. Antimicrob Agents Chemother. 2003;47(12):3973-3975.

28. Nilius AM, Shen LL, Hensey-Rudolff D, et al. In vitro antibacterial potency and spectrum of ABT-492, a new fluoroquinolone. Antimicrob Agents Chemother. 2003;47(10):3260-3269.

29. Almer LS, Hoffrage JB, Keller EL, Flamm RK, Shortridge VD. In vitro and bactericidal activities of ABT-492, a novel fluoroquinolone, against Gram-positive and Gram-negative organisms. Antimicrob Agents Chemother. 2004;48(7):2771-2777.

30. Lawrence L, Hopkins S, Sahm D, et al. Characterization and In vitro Activity of delafloxacin (DLX) against isolates from a phase 2 study of acute bacterial skin and skin structure infections (ABSSSI). Presented at: 52nd Interscience Conference on Antimicrobial Agents and Chemotherapy (ICAAC); September 9-12;2012; CA, USA (Abstract E-208).

31. Soge OO, Roberts MC, Lenderman C, et al. Evaluation of In vitro activity of delafloxacin against contemporary Neisseria gonorrhoeae isolates. Presented at: 54th Interscience Conference on Antimicrobial Agents and Chemotherapy; September 5-9;2014; CA, USA (Abstract C-1401).

32. Bauer J, Siala W, Tulkens PM, Van Bambeke F. A combined pharmacodynamic quantitative and qualitative model reveals the potent activity of daptomycin and delafloxacin against Satphylococcus aureus biofilms. Antimicrob Agents Chemother. 2013;57(6):2726-2737.

33. Siala W, Mingeot-Leclercq MP, Tulkens PM, Hallin M, Denis O, Van Bambeke F. Comparison of the antibiotic activities of daptomycin, vancomycin, and the investigational fluoroquinolone delafloxacin against biofilms from Staphylococcus aureus clinical isolates. Antimicrob Agents Chemother. 2014;58(11):6385-6397.

34. Soge OO, Salipante SJ, No D, Duffy E, Roberts MC. In vitro activity of Delafloxacin against clinical Neisseria gonorrhoeae isolates and selection of gonococcal delafloxacin resistance. Antimicrob Agents Chemother. 2016;60(5):3106-3111.

35. Hoover R, Hunt T, Benedict M, et al. Safety, tolerability and pharmacokinetic properties of intravenous delafloxacin after single and multiple doses in healthy volunteers. Clin Ther. 2016;38(1):53-65.

36. Hoover R, Hunt T, Benedict M, et al. Single and multiple ascendingdose studies of oral delafloxacin: effects of food, sex and age. Clin Ther. 2016;38(1):39-52.

37. Zinner SH, Vostrov SN, Alferova IV, Lubenko IY, Portnoy YA, Firsov AA. Comparative pharmacodynamics of the new fluoroquinolone ABT492 and ciprofloxacin with Escherichia coli and Pseudomonas aeruginosa in an in vitro dynamic model. Int $J$ Antimicrob Agents. 2004; 24(2):173-177.

38. Firsov AA, Vostrov SN, Lubenko IY, Arzamastsev AP, Portnoy YA, Zinner SH. ABT492 and levofloxacin: comparison of their pharmacodynamics and their abilities to prevent the selection of resistant Staphylococcus aureus in an in vitro dynamic model. J Antimicrob Chemother. 2004;54(1):178-186.

39. Firsov AA, Alferova IV, Smirnova MV, Lubenko IY, Portnoy YA, Zinner SH. Comparative pharmacodynamics of the new fluoroquinolone ABT492 and Levofloxacin with Streptococcus Pneumoniae in an in vitro dynamic model. Int J Antimicrob Agents. 2005;25(5):409-413.

40. Lepak AJ, Andes DR. In vivo pharmacodynamic target assessment of delafloxacin against staphylococcus aureus, Streptococcus pneumonia and Klebsiella pneumonia in the murine lung infection model. Antimicrob Agents Chemother. 2016;60(8):4764-4769.

41. Burak E, Bortolon E, Molstad D, et al. Pharmacokinetics and pharmacodynamics of delafloxacin in $S$. aureus murine thigh infection models. CA, USA, 12-15 September 2009 (Poster A1-1941).

42. O'Riordan W, Mehra P, Manos P, Kingsley J, Lawrence L, Cammarata S. A randomized phase 2 study comparing two doses of delafloxacin with tigecycline in adults with complicated skin and skin-structure infections. Int J Infect Dis. 2014;30:67-73.

43. Kingsley J, Mehra P, Lawrence LE, et al. A randomized, double-blind, phase 2 study to evaluate subjective and objective outcomes in patients with acute bacterial skin and skin structure infections treated with delafloxacin, linezolid or vancomycin. J Antimicrob Chemother. 2016;71(3):821-829.
44. Melinta Therapeutics, Inc. A phase 3, multicenter, randomized, doubleblind, active-controlled study to evaluate the efficacy and safety of delafloxacin compared with vancomycin + aztreonam in patients with acute bacterial skin and skin structure infections. Available from: https://clinicaltrials.gov/ct2/show/NCT01811732. NML Identifier: NCT01811732. Accessed March 15, 2017.

45. Melinta Therapeutics, Inc. A phase 3, multicenter, randomized, doubleblind, active controlled study to evaluate the efficacy + safety of IV + oral delafloxacin compared with vancomycin + aztreonam in patients with acute bacterial skin and skin structure infections. Available from: https:/clinicaltrials.gov/ct2/show/NCT01984684. NML Identifier: NCT01984684. Accessed March 15, 2017.

46. Longcor J, Hopkins S, Wikler M, Lawrence L. A phase 2 safety and effciacy study of oral delafloxacin in subjects with acute bacterial exacerbation of chronic bronchitis (ABECB). Presented at: ID Week; 2012; San Diego, CA. Available from: https://idsa.confex.com/idsa/2012/ webprogram/Paper37662. Accessed March 15, 2017.

47. Melinta Therapeutics, Inc. A phase 3, multicenter, randomized, doubleblind, comparator-controlled study to evaluate the safety and efficacy of intravenous to oral delafloxacin in adult subjects with communityacquired bacterial pneumonia. Available from: https://clinicaltrials.gov/ ct2/show/NCT02679573. NML Identifier: NCT02679573. Accessed March 15, 2017

48. Melinta Therapeutics, Inc. A comparative evaluation of the singledose efficacy of oral delafloxacin versus the single-dose efficacy of an intramuscular injection of ceftriaxone in subjects with uncomplicated urogenital gonorrhea. Available from: https://clinicaltrials.gov/ct2/ show/NCT02015637. NML Identifier NCT02015637. Accessed March 15, 2017.

49. Hoover R, Lawrence L, Smith C, Longcor J. Pharmacokinetics (PK) of delafloxacin in patients with varying degrees of renal impairment. Presented at: 53rd Interscience Conference on Antimicrobial Agents and Chemotherapy (ICAAC). CO, USA, 10-13 September 2013 (Abstract A-017E).

50. Hoover R, Marbury TC, Preston RA, et al. Clinical pharmacology of delafloxacin in patients with hepatic impairment. $J$ Clin Pharmacol. 2016;57(3):328-335.

51. Fuzi M. Dissimilar fitness associated with resistance to fluoroquinolones influences clonal dynamics of various multiresistant bacteria. Front Microbiol. 2016;7:1017.

52. FDA Drug Safety Communication: FDA advises restricting fluoroquinolone antibiotic use for certain uncomplicated infections; warns about disabling side effects than can occur together. Available from: https://www.fda.gov/Drugs/DrugSafety/ucm500143.htm. Accessed March 13, 2017.

53. Thabit AK, Crandon JL, Nicolau DP. Pharmacodynamic and pharmacokinetic profiling of delafloxacin in a murine lung model against community-acquired respiratory tract pathogens. Int J Antimicrob Agents. 2016;48(5):535-541.

54. Stass H, Rink AD, Delesen H, Kubitza D, Vestweber KH. Pharmacokinetics and peritoneal penetration of moxifloxacin in peritonitis. J Antimicrob Chemother. 2006;58(3):693-696.

55. Malangoni MA, Song J, Herrington J, Choudhri S, Pertel P. Randomized controlled trial of moxifloxacin compared with piperacillin-tazobactam and amoxicillin-clavulanate for the treatment of complicated intraabdominal infections. Ann Surg. 2006;244(2):2004-2211.

56. Weiss G, Reimnitz P, Hampel B, et al. Moxifloxacin for the treatment of patients with complicated intra-abdominal infections (the AIDA Study). J Chemother. 2009;21(2):170-180.

57. Goldstein EJ, Solomkin JS, Citron DM, Alder JD. Clinical efficacy and correlation of clinical outcomes with in vitro susceptibility for anaerobic bacteria in patients with complicated intra-abdominal infections treated with moxifloxacin. Clin Infect Dis. 2011;53(11):1074-1080.

58. Wagenlehner FM, Weidner W, Sörgel F, Nabe KG. The role of antibiotics in chronic bacterial prostatitis. Int J Antimicrob Agents. 2005;26(1):1-7.

59. Charalabopoulos K, Karachalios G, Baltogiannis D, Charalabopoulos A, Giannakopoulos X, Sofikitis N. Penetration of antimicrobial agents into the prostate. Chemotherapy. 2003;49(6):269-279. 
60. Lipsky BA, Giordano P, Choudhri S, Song J. Treating diabetic foot infections with sequential intravenous to oral moxifloxacin compared with piperacillin-tazobactam/amoxicillin-clavulanate. J Antimicrob Chemother. 2007;60(2):370-376.

61. Vick-Fragoso R, Hernández-Oliva G, Cruz-Alcázar J, et al. Efficacy and safety of sequential intravenous/oral moxifloxacin vs intravenous/ oral amoxicillin/clavulanate for complicated skin and skin structure infections. Infection. 2009;37(5):407-417.

62. Gyssens IC, Dryden M, Kujath P, et al. A randomized trial of the efficacy and safety of sequential intravenous/oral moxifloxacin monotherapy versus intravenous piperacillin/tazobactam followed by oral amoxicillin/ clavulanate for complicated skin and skin structure infections $J$ Antimicrob Chemother. 2011;66(11):2632-2642.

63. Majcher-Peszynska J, Sass M, Schipper S, et al. Pharmacokinetics and penetration of moxifloxacin into infected diabetic foot tissue in a large diabetic patient cohort. Eur J Clin Pharmacol. 2011;67(2):135-142.

64. Barberan J. Management of infections of osteoarticular prosthesis. Clin Microbiol Infect. 2006;12(Suppl 3):93-101.
65. Malincarne L, Ghebregzabher M, Moretti MV, et al. Penetration of moxifloxacin into bone in patients undergoing total knee arthroplasty. $J$ Antimicrob Chemother. 2006;57(5):950-954.

66. Metallidis S, Topsis D, Nikolaidis J, et al. Penetration of moxifloxacin and levofloxacin into cancellous and cortical bone in patients undergoing total hip arthroplasty. J Chemother. 2007;19(6):682-687.

67. Rimmele T, Boselli E, Breilh D, et al. Diffusion of levofloxacin into bone and synovial tissues. J Antimicrob Chemother. 2004;53(3):533-535.

68. Ishida H, Ishida Y, Kurosaka Y, Otani T, Sato K, Kobayashi H In vitro and in vivo activities of levofloxacin against biofilm-producing Pseudomonas aeruginosa. Antimicrob Agents Chemother. 1998;42(7): 1641-1645.

69. Roveta S, Schito AM, Marchese A, Schito GC. Activity of moxifloxacin on biofilms produced in vitro by bacterial pathogens involved in acute exacerbations of chronic bronchitis. Int J Antimicrob Agents. 2007; 30(5):415-421.

\section{Publish your work in this journal}

Drug Design, Development and Therapy is an international, peerreviewed open-access journal that spans the spectrum of drug design and development through to clinical applications. Clinical outcomes, patient safety, and programs for the development and effective, safe, and sustained use of medicines are the features of the journal, which has also been accepted for indexing on PubMed Central. The manuscript management system is completely online and includes a very quick and fair peer-review system, which is all easy to use. Visit http://www.dovepress.com/testimonials.php to read real quotes from published authors.

Submit your manuscript here: http://www.dovepress.com/drug-design-development-and-therapy-journal 